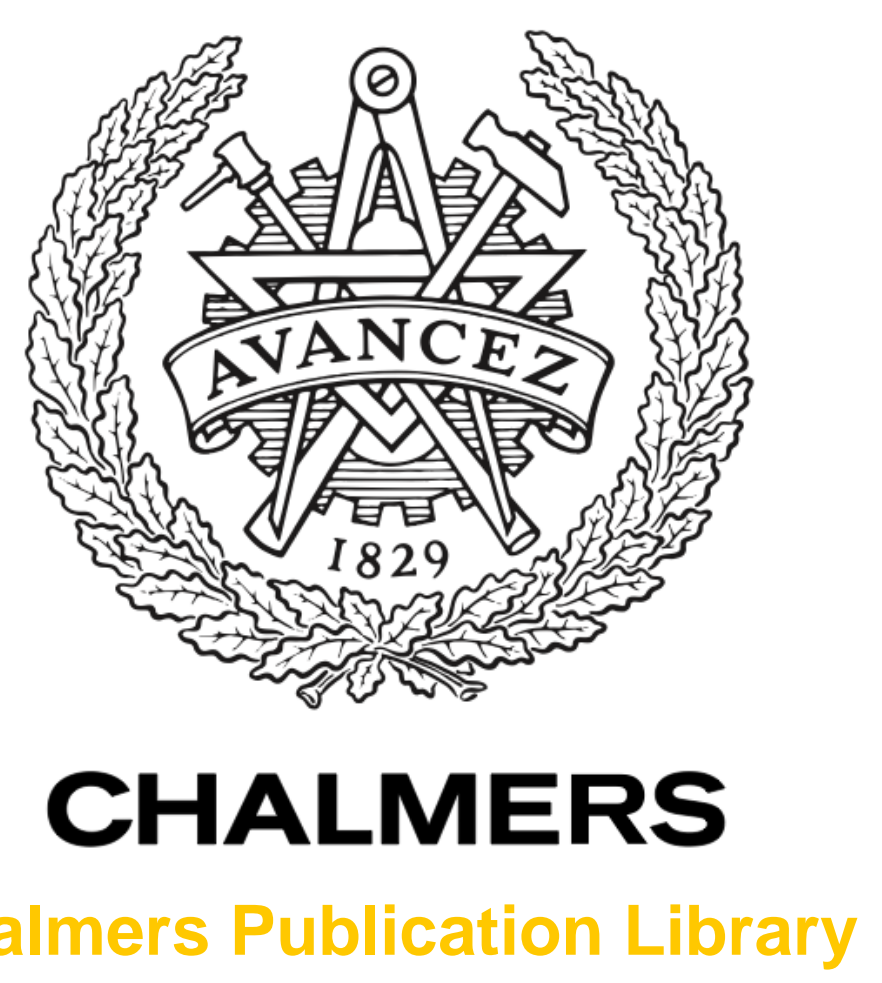

Chalmers Publication Library

\title{
On the Performance of HARQ-Based RF-FSO Links
}

This document has been downloaded from Chalmers Publication Library (CPL). It is the author's version of a work that was accepted for publication in:

\section{GLOBECOM2015}

Citation for the published paper:

Makki, B. ; Svensson, T. ; Eriksson, T. et al. (2015) "On the Performance of HARQ-Based RF-FSO Links". GLOBECOM2015

Downloaded from: http://publications.lib.chalmers.se/publication/234018

Notice: Changes introduced as a result of publishing processes such as copy-editing and formatting may not be reflected in this document. For a definitive version of this work, please refer to the published source. Please note that access to the published version might require a subscription. 


\title{
On the Performance of HARQ-based RF-FSO Links
}

\author{
Behrooz Makki*, Tommy Svensson*, Thomas Eriksson* and Mohamed-Slim Alouini ${ }^{\dagger}$ \\ *Department of Signals and Systems, Chalmers University of Technology, Gothenburg, Sweden \\ \{behrooz.makki, tommy.svensson, thomase\}@chalmers.se \\ ${ }^{\dagger}$ Computer, Electrical, and Mathematical Science and Engineering Division, \\ King Abdullah University of Science and Technology (KAUST), Thuwal, Saudi Arabia, slim.alouini@kaust.edu.sa
}

\begin{abstract}
This paper studies the performance of the hybrid radio-frequency (RF) and free-space optical (FSO) links assuming perfect channel state information (CSI) at the receiver. Considering the cases with and without hybrid automatic repeat request (HARQ), we derive closed-form expressions for the message decoding probabilities as well as the throughput and the outage probability of the RF-FSO setups. We also evaluate the effect of different channel conditions on the throughput and the outage probability. The results show the efficiency of the RF-FSO links in different conditions.
\end{abstract}

\section{INTRODUCTION}

The next generation communication networks must provide high-rate reliable data streams. To address the demands, the combination of different techniques are considered, among which free-space optical (FSO) communication is very promising [1], [2]. FSO systems provide fiber-like data rates through the atmosphere using lasers or light emitting diodes (LEDs). Thus, the FSO setups can be used for a wide range of applications such as last-mile access, fiber back-up, back-haul for wireless cellular networks, and disaster recovery. However, such links are highly susceptible to atmospheric effects and, consequently, are unreliable. An efficient method to improve the reliability in FSO systems is to rely on an additional radiofrequency $(\mathrm{RF})$ link to create a hybrid RF-FSO communication system.

Typically, to achieve data rates comparable to those in the FSO link, a millimeter wavelength carrier is selected for the $\mathrm{RF}$ link. As a result, the RF link is also subject to atmospheric effects such as rain and scintillation. However, a good point is that these links are complementary because the RF (resp. the FSO) signal is severely attenuated by rain (resp. fog/cloud) while the FSO (resp. the RF) signal is not. Therefore, the link reliability and the service availability are considerably improved via joint RF-FSO based data transmission.

The performance of RF-FSO systems is studied in different papers, e.g., [2]-[8], where the RF and the FSO links are considered as separate links and the RF link acts as a backup when the FSO link is down. In the meantime, there are works such as [9]-[14] in which the RF and the FSO links are combined to improve the system performance. Moreover, the implementation of hybrid automatic repeat request (HARQ) in RF-based (resp. FSO-based) systems is investigated in, e.g., [15]-[21] (resp. [22]-[27]), while the HARQ-based RF-FSO systems have been rarely studied, e.g., [14], [28].

978-1-4799-5863-4/14/\$31.00 (C)2014 IEEE

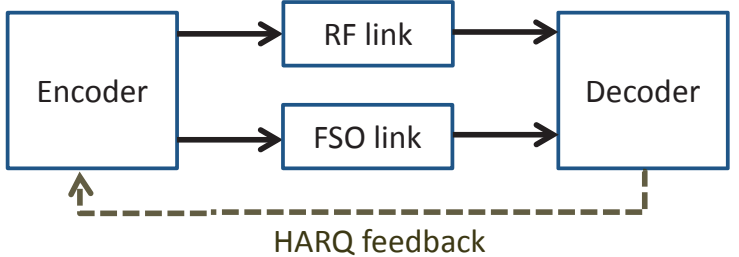

Figure 1. Channel model. The data is jointly transmitted by the RF and the FSO links and, in each round of HARQ, the receiver decodes the data based on all received signals.

In this paper, we study the data transmission efficiency of RF-FSO systems from an information theoretic point of view. We derive closed-form expressions for the message decoding probabilities as well as the system throughput and outage probability (Lemmas 1-3). The results are obtained in the cases with and without HARQ. Also, we investigate the effect of different channel conditions on the performance of RF-FSO setups and compare the results with the cases utilizing either the RF or the FSO link separately. Note that, while the results are presented for the RF-FSO setups, the same analysis as in the paper is useful for other coordinated data transmission schemes as well (see Section IV for more discussions).

As opposed to [2]-[8], we consider joint data transmission/reception in the RF and FSO links. Also, the paper is different from [9]-[28] because we study the performance of HARQ in joint RF-FSO links and derive new analyti$\mathrm{cal} /$ numerical results on the message decoding probabilities, and outage probability/throughput which have not been presented before.

The numerical and the analytical results show that the joint implementation of the RF and FSO links leads to substantial performance improvement, compared to the cases with only the RF or the FSO link. For instance, consider the exponential distribution and the common relative coherence times of the $\mathrm{RF}$ and FSO links. Then, with the initial code rate $R=5$ natsper-channel-use (npcu), a maximum of $M=2$ retransmission rounds of the HARQ and the outage probability $10^{-2}$, the joint RF-FSO based data transmission reduces the required power by 16 and $4 \mathrm{~dB}$, compared to the cases with only the RF or the FSO link, respectively (see Fig. 6 for more details).

\section{System ModeL}

Consider a joint RF-FSO system, as demonstrated in Fig. 1. The data sequence is encoded into parallel FSO and RF bit streams. The FSO link employs intensity modulation and direct 
detection while the RF link modulates the encoded bits and up-converts the baseband signal to a millimeter wavelength RF carrier frequency. Then, the FSO and the RF signals are simultaneously sent to the receiver. At the receiver, the received RF (FSO) signal is down-converted to baseband (resp. collected by an aperture and converted to an electrical signal via photo-detection) and the signals are sent to the decoder which decodes the received signals jointly. Finally, we assume perfect synchronization between the links.

The channel coefficients of the RF and the FSO links are assumed to be known at the receiver which is an acceptable assumption in block-fading conditions [15]-[24]. We then assume no channel state information (CSI) feedback to the transmitter, except for the HARQ feedback bits. The feedback channel is supposed to be delay- and error-free.

Let us define a packet as the transmission of a codeword along with all its possible retransmissions. As the most promising HARQ approach leading to highest throughput/lowest outage probability [16]-[21], we consider the incremental redundancy (INR) HARQ with a maximum of $M$ retransmissions, i.e., the message is retransmitted a maximum of $M$ times. Using INR HARQ, $K$ information nats are encoded into a parent codeword of length $M L$ channel uses. The parent codeword is then divided into $M$ sub-codewords of length $L$ channel uses which are sent in the successive transmission rounds. Thus, the equivalent data rate, i.e., the code rate, at the end of round $m$ is $\frac{K}{m L}=\frac{R}{m}$ where $R=\frac{K}{L}$ denotes the initial code rate. In each round, the receiver combines all received sub-codewords to decode the message. The retransmission continues until the message is correctly decoded or the maximum permitted transmission round is reached. Note that setting $M=1$ represents the cases without HARQ, i.e., open-loop communication.

\section{SUMMARY OF THE PAPER}

It has been previously showed that for different channel models, the throughput and the outage probability of different HARQ protocols can be written as [16]-[19]

$$
\eta=R \frac{1-\operatorname{Pr}\left(W_{M} \leq \frac{R}{M}\right)}{1+\sum_{m=1}^{M-1} \operatorname{Pr}\left(W_{m} \leq \frac{R}{m}\right)}
$$

and

$$
\operatorname{Pr}(\text { Outage })=\operatorname{Pr}\left(W_{M} \leq \frac{R}{M}\right),
$$

respectively, where $W_{m}$ is the accumulated mutual information (AMI) at the end of round $m$. Also, $\operatorname{Pr}\left(W_{m} \leq \frac{R}{m}\right)$ denotes the probability that the data is not correctly decoded up to the end of the $m$-th round. In this way, the throughput and the outage probability of HARQ protocols are monotonic functions of the probabilities $\operatorname{Pr}\left(W_{m} \leq \frac{R}{m}\right), \forall m$. This is because the system performance depends on the retransmission round in which the codewords are correctly decoded. Moreover, the probability $\operatorname{Pr}\left(W_{m} \leq \frac{R}{m}\right)$ is directly linked to the AMI $W_{m}$ which is a random variable and function of the channel realizations experienced in rounds $n=1, \ldots, m$. As such, to analyze the throughput and the outage probability, the key

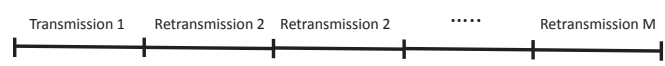

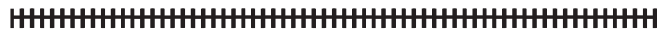

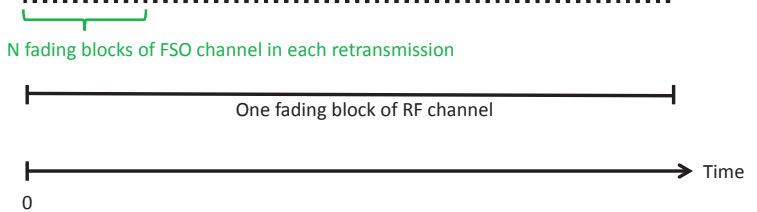

Figure 2. Time scales. The RF link is supposed to remain constant in the retransmissions (quasi-static channel [17], [18], [20], [21]) while in each retransmission round of HARQ $N$ different channel realizations are experienced in the FSO link.

point is to determine the AMIs as functions of channel realization(s) and find their corresponding cumulative distribution functions (CDFs) ${ }^{1} \quad F_{W_{m}}, m=1, \ldots, M$. Then, having the CDFs, the probabilities $\operatorname{Pr}\left(W_{m} \leq \frac{R}{m}\right)$ and, consequently, the considered performance metrics are obtained. Therefore, instead of concentrating on (1)-(2), we first find the CDFs $F_{W_{m}}, m=1, \ldots, M$, for the INR-based RF-FSO system.

To find the CDFs, we utilize the properties of the RF and the FSO links to derive the AMIs as in (3). Since there is no closed-form expression for the CDFs of the AMIs, we need to use different approximation techniques. In this paper, we use the central limit Theorem (CLT) to approximate the contribution of the FSO link on the AMI by an equivalent Gaussian random variable. Using the CLT, we find the mean and the variance of the equivalent random variable for the exponential and log-normal distributions of the FSO link as given in (4)-(5) and (6)-(9), respectively. With the derived means and variances of the Gaussian variable, we find the CDF of the AMIs in Lemmas 1-3 (see Section IV.A for details). In Section V, we validate the accuracy of the approximations and evaluate the throughput/outage probability of the RF-FSO system for different channel conditions. Finally, note that, due to space limits, this paper concentrates on the exponential and log-normal distributions of the FSO link, while performance analysis in the cases with Gamma-Gamma PDF of the FSO link is presented in the extended version of the paper [29].

\section{AnAlytical RESUlts}

In RF-FSO systems, it was demonstrated by, e.g., [10], [30][32], that the RF link experiences very slow variations and the coherence time of the RF link is in the order of $10^{2}-10^{3}$ times larger than the coherence time of the FSO link. Here, we consider the setup as illustrated in Fig. 2 where the RF link remains constant in the retransmissions (quasi-static channel [17], [18], [20], [21]) while in each retransmission round of HARQ $N$ different channel realizations are experienced in the FSO link. However, note that this is not a necessary condition because 1) the same analysis holds for the cases with shorter coherence time of the RF link, compared to the coherence time of the FSO link and 2) as presented in [29], we can derive the

\footnotetext{
${ }^{1}$ The $\mathrm{CDF}$ and the probability distribution function (PDF) of a random
} variable $X$ are denoted by $F_{X}($.$) and f_{X}($.$) , respectively.$ 
results in the cases with few, possibly 1, channel realizations of the FSO link during the packet transmission.

Considering Fig. 2, we can use the results of [33, Chapter 7] and [34, Chapter 15] to find the AMI of the joint RF-FSO link at the end of the $m$-th round as

$$
\begin{aligned}
& W_{m}=\log \left(1+P_{\mathrm{RF}} G_{\mathrm{RF}}\right) \\
& \quad+\frac{1}{m} \sum_{j=1}^{m}\left(\frac{1}{N} \sum_{i=1}^{N} \log \left(1+P_{\mathrm{FSO}} G_{\mathrm{FSO}, 1+(j-1) i}\right)\right) \\
& =\log \left(1+P_{\mathrm{RF}} G_{\mathrm{RF}}\right)+\mathcal{Y}_{(m, N)}, \\
& \mathcal{Y}_{(m, N)} \doteq \frac{1}{m N} \sum_{j=1}^{m} \sum_{i=1}^{N} \log \left(1+P_{\mathrm{FSO}} G_{\mathrm{FSO}, 1+(j-1) i}\right) .
\end{aligned}
$$

Here, $P_{\mathrm{RF}}$ and $P_{\mathrm{FSO}}$ are, respectively, the transmission powers in the RF and FSO links. Also, $G_{\mathrm{RF}}$ and $G_{\mathrm{FSO}, j}$ 's denote the channel gain realizations of the RF and the FSO links in different retransmission rounds, respectively.

Considering the conventional channel conditions of the RF and FSO links and different values of $N$, there is no closedform expression for the CDF of $W_{m}, \forall m$. Therefore, we use the CLT to approximate $\mathcal{Y}_{(m, N)}$ by the Gaussian random variable $\mathcal{Z} \sim \mathcal{N}\left(\mu, \frac{1}{m N} \sigma^{2}\right)$ where $\mu$ and $\sigma^{2}$ are the mean and variance determined based on the FSO link channel condition.

Reviewing the literature and depending on the channel condition, the FSO link is commonly considered to follow exponential, log-normal or Gamma-Gamma distributions, e.g., [10], [22], [35]. We present the performance analysis for the Gamma-Gamma PDF of the FSO link in [29]. For the exponential distribution of the FSO link, i.e., $f_{G_{\mathrm{FSO}}}(x)=\lambda e^{-\lambda x}$ with $\lambda$ being the long-term channel coefficient, we have

$$
\begin{gathered}
\mu=E\left\{\log \left(1+P_{\mathrm{FSO}} G_{\mathrm{FSO}}\right)\right\}=\int_{0}^{\infty} f_{G_{\mathrm{FSO}}}(x) \log \left(1+P_{\mathrm{FSO}} x\right) \mathrm{d} x \\
\stackrel{(a)}{=} P_{\mathrm{FSO}} \int_{0}^{\infty} \frac{1-F_{G_{\mathrm{FSO}}}(x)}{1+P_{\mathrm{FSO}} x} \mathrm{~d} x=-e^{\frac{\lambda}{P_{\mathrm{FSO}}}} \mathrm{Ei}\left(-\frac{\lambda}{P_{\mathrm{FSO}}}\right)
\end{gathered}
$$

and $\sigma^{2}=\rho^{2}-\mu^{2}$ with

$$
\begin{aligned}
& \rho^{2}=E\left\{\log \left(1+P_{\mathrm{FSO}} G_{\mathrm{FSO}}\right)^{2}\right\} \\
& =\int_{0}^{\infty} f_{G_{\mathrm{FSO}}}(x) \log ^{2}\left(1+P_{\mathrm{FSO}} x\right) \mathrm{d} x \\
& \stackrel{(b)}{=} 2 P_{\mathrm{FSO}} \int_{0}^{\infty} \frac{e^{-\lambda x}}{1+P_{\mathrm{FSO}} x} \log \left(1+P_{\mathrm{FSO}} x\right) \mathrm{d} x \\
& \stackrel{(c)}{\simeq} 2 P_{\mathrm{FSO}}^{2} \int_{0}^{\beta} \frac{x e^{-\lambda x}}{1+P_{\mathrm{FSO}} x} \mathrm{~d} x \\
& +2 \sum_{n=1}^{\infty} \frac{(-1)^{n+1}\left(P_{\mathrm{FSO}}\right)^{n}}{n} \int_{\beta}^{\infty} e^{-\lambda x} x^{n-1} \mathrm{~d} x \\
& =2\left(e^{\frac{\lambda}{P_{\mathrm{FSO}}}}\left(\operatorname{Ei}\left(\frac{-\lambda}{P_{\mathrm{FSO}}}\right)-\mathrm{Ei}\left(-\beta \lambda-\frac{\lambda}{P_{\mathrm{FSO}}}\right)\right)\right. \\
& \left.+\frac{P_{\mathrm{FSO}}}{\lambda}\left(1-e^{-\beta \lambda}\right)\right) \\
& +2 \sum_{n=1}^{\infty} \frac{(-1)^{n+1}}{n}\left(\frac{P_{\mathrm{FSO}}}{\lambda}\right)^{n} \Gamma(n, \beta \lambda), \forall \beta .
\end{aligned}
$$

Here, $E\{$.$\} denotes the expectation operator. Also, (a)$ and $(b)$ are obtained by partial integration. Then, $(c)$ consists of two integration parts where the first one comes from $\log (1+x)=x$ for small $x$ 's, and the second one is obtained by Taylor expansion of the logarithmic term and the approximation $\frac{1}{1+P_{\mathrm{FSO}} x} \simeq \frac{1}{P_{\mathrm{FSO}} x}$ for high values of $P_{\mathrm{FSO}}$ and $x \geq \beta$. Note that the approximation is very tight for small values of $\beta$. Finally, the last equalities in (4) and (5) follow from the definition of the exponential integral function $\operatorname{Ei}(x)=\int_{x}^{\infty} \frac{e^{-t} \mathrm{~d} t}{t}$ and the incomplete Gamma function $\Gamma(s, x)=\int_{x}^{\infty} t^{s-1} e^{-t} \mathrm{~d} t$.

For the log-normal distribution of the FSO link, i.e., $f_{G_{\mathrm{FSO}}}(x)=\frac{1}{\sqrt{2 \pi} \delta x} e^{-\frac{(\log (x)-\varpi)^{2}}{2 \delta^{2}}}$ where $\delta$ and $\varpi$ represent the long-term channel parameters, the mean $\mu$ is rephrased as

$$
\begin{aligned}
\mu= & P_{\mathrm{FSO}} \int_{0}^{\infty} \frac{1-F_{G_{\mathrm{FSO}}}(x)}{1+P_{\mathrm{FSO}} x} \mathrm{~d} x \\
\stackrel{(d)}{=} & \frac{P_{\mathrm{FSO}}}{2} \int_{0}^{\infty} \frac{1-\operatorname{erf}\left(\frac{\log (x)-\varpi}{\sqrt{2} \delta}\right)}{1+P_{\mathrm{FSO}} x} \mathrm{~d} x \\
= & P_{\mathrm{FSO}} \int_{0}^{\infty} \frac{Q\left(\frac{\log (x)-\varpi}{\delta}\right)}{1+P_{\mathrm{FSO}} x} \mathrm{~d} x \stackrel{(e)}{\simeq} P_{\mathrm{FSO}} \int_{0}^{\infty} \frac{U(x)}{1+P_{\mathrm{FSO}} x} \mathrm{~d} x \\
= & P_{\mathrm{FSO}}\left(\int_{0}^{\max \left(0, \frac{1}{2 c}+e^{\varpi}\right)} \frac{1}{1+P_{\mathrm{FSO}} x} \mathrm{~d} x\right. \\
& \left.+\int_{\max \left(0, \frac{1}{2 c}+e^{\varpi}\right)}^{\frac{-1}{2 c}+e^{\varpi}+c\left(x-e^{\varpi}\right)} \mathrm{d} x\right) \\
= & \log \left(1+P_{\mathrm{FSO}} x\right. \\
& +\left(\frac{1}{2}-c e^{\varpi}-\frac{c}{P_{\mathrm{FSO}}}\right) \log \left(\frac{1}{1+P_{\mathrm{FSO}} \max \left(0, \frac{1}{2 c}+e^{\varpi}\right)}\right) \\
& +c\left(\frac{-1}{2 c}+e^{\varpi}-\max \left(0, \frac{1}{2 c}+e^{\varpi}\right)\right), c \doteq \frac{-e^{-\varpi}}{\delta \sqrt{2 \pi}} .
\end{aligned}
$$

Here, $\operatorname{erf}(x)=\frac{2}{\sqrt{\pi}} \int_{0}^{x} e^{-t^{2}} \mathrm{~d} t$ and $Q(x)=\frac{1}{\sqrt{2 \pi}} \int_{x}^{\infty} e^{-\frac{t^{2}}{2}} \mathrm{~d} t$ represent the error and the Gaussian $Q$ functions, respectively. Moreover, $(d)$ holds for the log-normal distribution and $(e)$ comes from the linearization technique

$$
\begin{aligned}
& Q\left(\frac{\log (x)-\varpi}{\delta}\right) \simeq U(x) \\
& U(x)=\left\{\begin{array}{cc}
1 & x<\frac{1}{2 c}+e^{\varpi}, \\
\frac{1}{2}+c\left(x-e^{\varpi}\right) & x \in\left[\begin{array}{c}
\frac{1}{2 c}+e^{\varpi}, \frac{-1}{2 c}+e^{\varpi} \\
0
\end{array}\right. \\
0 & x \frac{-1}{2 c}+e^{\varpi},
\end{array}\right.
\end{aligned}
$$

with

$$
c=\left.\frac{\partial\left(Q\left(\frac{\log (x)-\varpi)}{\sqrt{2} \delta}\right)\right)}{\partial x}\right|_{x=e^{\varpi}}=\frac{-e^{-\varpi}}{\delta \sqrt{2 \pi}}
$$

which is found by the derivative of $Q\left(\frac{\log (x)-\varpi}{\sqrt{2} \delta}\right)$ at point $x=e^{\varpi}$. Also, following the same procedure as in (6), the 
variance $\sigma^{2}$ is determined as

$$
\begin{aligned}
& \sigma^{2}=\rho^{2}-\mu^{2}, \\
& \rho^{2}=2 P_{\mathrm{FSO}} \int_{0}^{\infty} \frac{\log \left(1+P_{\mathrm{FSO}} x\right)}{1+P_{\mathrm{FSO}} x} Q\left(\frac{\log (x)-\varpi}{\delta}\right) \mathrm{d} x \\
& \stackrel{(f)}{\simeq} 2 P_{\mathrm{FSO}} \int_{0}^{\infty} \frac{\log \left(1+P_{\mathrm{FSO}} x\right)}{1+P_{\mathrm{FSO}} x} U(x) \mathrm{d} x \\
& =\left(\log \left(1+P_{\mathrm{FSO} \max }\left(0, \frac{1}{2 c}+e^{\varpi}\right)\right)\right)^{2}+ \\
& \frac{1}{P_{\mathrm{FSO}}}\left(( ( \frac { 1 } { 2 } - c e ^ { \varpi } ) P _ { \mathrm { FSO } } - c ) \left(\left(\log \left(1+P_{\mathrm{FSO}}\left(\frac{-1}{2 c}+e^{\varpi}\right)\right)\right)^{2}\right.\right. \\
& -2 c P_{\mathrm{FSO}}\left(\frac{-1}{2 c}+e^{\varpi}-\max \left(0, \frac{1}{2 c}+e^{\varpi}\right)\right) \\
& +2 c\left(\left(1+P_{\mathrm{FSO}}\left(\frac{-1}{2 c}+e^{\varpi}\right)\right) \log \left(1+P_{\mathrm{FSO}}\left(\frac{-1}{2 c}+e^{\varpi}\right)\right)\right. \\
& -\left(1+P_{\mathrm{FSO}} \max \left(0, \frac{1}{2 c}+e^{\varpi}\right)\right) \times \\
& \left.\left.\log \left(1+P_{\mathrm{FSO}} \max \left(0, \frac{1}{2 c}+e^{\varpi}\right)\right)\right)\right)
\end{aligned}
$$

for the log-normal distribution of the FSO link, where $(f)$ comes from (7)-(8).

Having $\mu$ and $\sigma^{2}$, we find the CDFs $F_{W_{m}}, \forall m$, as follows. Consider Rayleigh-fading conditions for the RF link where the fading coefficients follow $H_{\mathrm{RF}} \sim \mathcal{C N}(0,1)$ and, consequently, $f_{G_{\mathrm{RF}}}(x)=e^{-x}, G_{\mathrm{RF}}=\left|H_{\mathrm{RF}}\right|^{2}$. Using (3) and the mean and variance of $\mathcal{Z}$, the CDFs of the AMIs are given by

$$
\begin{aligned}
& F_{W_{m}}(u)=\operatorname{Pr}\left(\log \left(1+P_{\mathrm{RF}} G_{\mathrm{RF}}\right)+\mathcal{Y}_{(m, N)} \leq u\right) \\
& =\int_{0}^{\frac{e^{u}-1}{P_{\mathrm{RF}}}} f_{G_{\mathrm{RF}}}(x) \operatorname{Pr}\left(\mathcal{Y}_{(m, N)} \leq u-\log \left(1+P_{\mathrm{RF}} G_{\mathrm{RF}}\right)\right) \mathrm{d} x \\
& \stackrel{(g)}{=} \int_{0}^{\frac{e^{u}-1}{P_{\mathrm{RF}}}} e^{-x} Q\left(\frac{\sqrt{m N}\left(\log \left(1+P_{\mathrm{RF}} x\right)+\mu-u\right)}{\sigma}\right) \mathrm{d} x, \forall m, N
\end{aligned}
$$

where $(g)$ comes from the CDF of Gaussian distributions and CLT. Also, for the exponential and log-normal distribution of the FSO link the mean and variance $\left(\mu, \sigma^{2}\right)$ are given by (4)-(5) and (6)-(9), respectively. Therefore, the final step to derive the throughput and the outage probability is to find (10) while it does not have closed-form expression. The following lemmas propose several approximation/bounding approaches for the CDF of the AMIs and, consequently, the throughput/outage probability.

Lemma 1: The throughput and the outage probability of the HARQ-based RF-FSO setup are approximately given by

$$
\eta=R \frac{1-\mathcal{F}\left(\frac{R}{M}\right)}{1+\sum_{m=1}^{M-1} \mathcal{F}\left(\frac{R}{m}\right)}
$$

and

$$
\operatorname{Pr}(\text { Outage })=\mathcal{F}\left(\frac{R}{M}\right),
$$

respectively, with $\mathcal{F}(x)$ defined in (14) on top of the next page.

Proof. To find the approximations, we implement $Q\left(\frac{\sqrt{m N}\left(\log \left(1+P_{\mathrm{RF}} x\right)+\mu-u\right)}{\sigma}\right) \simeq V(x)$ with

$$
\begin{aligned}
& V(x)=\left\{\begin{array}{cc}
1 & x<\chi_{1}, \\
\frac{1}{2}+\frac{\sqrt{m N} P_{\mathrm{RF}} e^{\mu-u}\left(x-\frac{e^{u-\mu}-1}{P_{\mathrm{RF}}}\right)}{\sigma \sqrt{2 \pi}} & x \in\left[\chi_{1}, \chi_{2}\right], \\
0 & x>\chi_{2},
\end{array}\right. \\
& \chi_{1} \doteq \frac{-\sigma \sqrt{\pi} e^{u-\mu}}{P_{\mathrm{RF}} \sqrt{2 m N}}+\frac{e^{u-\mu}-1}{P_{\mathrm{RF}}}, \chi_{2} \doteq \frac{\sigma \sqrt{\pi} e^{u-\mu}}{P_{\mathrm{RF}} \sqrt{2 m N}}+\frac{e^{u-\mu}-1}{P_{\mathrm{RF}}}
\end{aligned}
$$

in (10), which leads to (14) on top of the next page. Here, $V(x)$ is obtained by applying the same linearization technique as in (7) on the Gaussian $Q$ function of (10) at point $x=\frac{e^{u-\mu}-1}{P_{\mathrm{RF}}}$. Then, using (14) in (1)-(2), one can find the throughput and outage probability, as stated in the lemma.

Along with the approximation scheme of Lemma 1, Lemmas 2-3 derive upper and lower bounds of the system performance assuming that the mean and variance of the equivalent Gaussian random variable $\mathcal{Z}$ are calculated accurately.

Lemma 2: The performance of the RF-FSO system is upperestimated, i.e., the throughput is upper bounded and the outage probability is lower bounded, via the following inequality

$$
F_{W_{m}}(u) \geq \mathcal{V}(u),
$$

with $\mathcal{V}(u)$ given in (16) on top of the next page.

Proof. As mentioned before and in [16]-[19], the throughput (resp. the outage probability) of the HARQ-based systems is a decreasing (resp. increasing) function of the probabilities $F_{W_{m}}\left(\frac{R}{m}\right), \forall m$ (resp. $F_{W_{M}}\left(\frac{R}{M}\right)$ ). Thus, the throughput (resp. the outage probability) is upper bounded (resp. lower bounded) by lower bounding $F_{W_{m}}(),. \forall m$. On the other hand, because the $Q$ function is a decreasing function and $r(x)=$ $\frac{\sqrt{m N}\left(\log \left(1+P_{\mathrm{RF}} x\right)+\mu-u\right)}{\sigma}$ is concave in $x$, the CDFs of the AMIs are lower bounded if $r(x)$ is replaced by its first order Taylor expansion at any point. Considering the Taylor expansion of $r(x)$ at $x=\frac{e^{u-\mu}-1}{P_{\mathrm{RF}}}$, we can bound the probabilities as in (16) on top of the next page.

Here, $(h)$ comes from partial integration and $\frac{\mathrm{d} Q(y(x))}{\mathrm{d} x}=$ $\frac{-1}{\sqrt{2 \pi}} \frac{\mathrm{d} y}{\mathrm{~d} x} e^{-\frac{y^{2}(x)}{2}}$. Also, the last equality is obtained by some manipulations and the definition of the error function.

Lemma 3: An under-estimate of the performance of the RF-FSO system is given by

$$
F_{W_{m}}(u) \leq \mathcal{T}(u),
$$

where $\mathcal{T}(u)$ is defined in (18).

Proof. To derive an under-estimate of the system performance, i.e., a lower bound of the throughput and an upper bound of the 


$$
\begin{aligned}
& F_{W_{m}}(u) \simeq \int_{0}^{\frac{e^{u}-1}{P_{\mathrm{RF}}}} e^{-x} V(x) \mathrm{d} x=\int_{0}^{\max \left(0, \chi_{1}\right)} e^{-x} \mathrm{~d} x+\int_{\max \left(0, \chi_{1}\right)}^{\min \left(\chi_{2}, \frac{e^{u}-1}{P_{\mathrm{RF}}}\right)} e^{-x}\left(\frac{1}{2}+\frac{\sqrt{m N} P_{\mathrm{RF}} e^{\mu-u}\left(x-\frac{e^{u-\mu}-1}{P_{\mathrm{RF}}}\right)}{\sigma \sqrt{2 \pi}}\right) \mathrm{d} x \\
& =1-e^{-\max \left(0, \frac{-\sigma \sqrt{\pi} e^{u-\mu}}{P_{\mathrm{RF}} \sqrt{2 m N}}+\frac{e^{u-\mu}-1}{P_{\mathrm{RF}}}\right)}+\left(\frac{1}{2}+\frac{\sqrt{m N}\left(e^{\mu-u}-1\right)}{\sigma \sqrt{2 \pi}}\right)\left(e^{-\max \left(0, \frac{-\sigma \sqrt{\pi} e^{u-\mu}}{P_{\mathrm{RF}} \sqrt{2 m N}}+\frac{e^{u-\mu}-1}{P_{\mathrm{RF}}}\right)}-e^{-\min \left(\frac{\sigma \sqrt{\pi} e^{u-\mu}}{P_{\mathrm{RF}} \sqrt{2 m N}}+\frac{e^{u-\mu}-1}{P_{\mathrm{RF}}}, \frac{e^{u}-1}{P_{\mathrm{RF}}}\right)}\right) \\
& +\frac{\sqrt{m N} P_{\mathrm{RF}} e^{\mu-u}}{\sigma \sqrt{2 \pi}}\left(\left(1+\max \left(0, \frac{-\sigma \sqrt{\pi} e^{u-\mu}}{P_{\mathrm{RF}} \sqrt{2 m N}}+\frac{e^{u-\mu}-1}{P_{\mathrm{RF}}}\right)\right) e^{-\max \left(0, \frac{-\sigma \sqrt{\pi} e^{u-\mu}}{P_{\mathrm{RF}} \sqrt{2 m N}}+\frac{e^{u-\mu}-1}{P_{\mathrm{RF}}}\right)}\right. \\
& \left.-\left(1+\min \left(\frac{\sigma \sqrt{\pi} e^{u-\mu}}{P_{\mathrm{RF}} \sqrt{2 m N}}+\frac{e^{u-\mu}-1}{P_{\mathrm{RF}}}, \frac{e^{u}-1}{P_{\mathrm{RF}}}\right)\right) e^{-\min \left(\frac{\sigma \sqrt{\pi} e^{u-\mu}}{P_{\mathrm{RF}} \sqrt{2 m N}}+\frac{e^{u-\mu}-1}{P_{\mathrm{RF}}}, \frac{e^{u}-1}{P_{\mathrm{RF}}}\right)}\right)=\mathcal{F}(u) . \\
& F_{W_{m}}(u) \geq \int_{0}^{\frac{e^{u}-1}{P_{\mathrm{RF}}}} e^{-x} Q\left(\frac{P_{\mathrm{RF}} \sqrt{m N} e^{\mu-u}}{\sigma}\left(x-\frac{e^{u-\mu}-1}{P_{\mathrm{RF}}}\right)\right) \mathrm{d} x \stackrel{(h)}{=} Q\left(\frac{\sqrt{m N}\left(e^{\mu-u}-1\right)}{\sigma}\right)-e^{-\frac{e^{u}-1}{P_{\mathrm{RF}}}} Q\left(\frac{P_{\mathrm{RF}} \sqrt{m N} e^{\mu-u}}{\sigma}\left(\frac{e^{u}-1}{P_{\mathrm{RF}}}-\frac{e^{u-\mu}-1}{P_{\mathrm{RF}}}\right)\right) \\
& -\frac{P_{\mathrm{RF}} \sqrt{m N} e^{\mu-u}}{\sigma \sqrt{2 \pi}} \int_{0}^{\frac{e^{u}-1}{P_{\mathrm{RF}}}} e^{-\left(x+\frac{P_{\mathrm{RF}}^{2} m N e^{2(\mu-u)}}{2 \sigma^{2}}\left(x-\frac{e^{u-\mu}-1}{P_{\mathrm{RF}}}\right)^{2}\right)} \mathrm{d} x=Q\left(\frac{\sqrt{m N}\left(e^{\mu-u}-1\right)}{\sigma}\right)-e^{-\frac{e^{u}-1}{P_{\mathrm{RF}}}} Q\left(\frac{P_{\mathrm{RF}} \sqrt{m N} e^{\mu-u}}{\sigma}\left(\frac{e^{u}-1}{P_{\mathrm{RF}}}-\frac{e^{u-\mu}-1}{P_{\mathrm{RF}}}\right)\right) \\
& +\frac{1}{2} e^{\frac{\sigma^{2}}{2 P_{\mathrm{RF}}^{2} m N e^{2(\mu-u)}}-\frac{e^{u-\mu}-1}{P_{\mathrm{RF}}}}\left(\operatorname{erf}\left(\frac{\frac{P_{\mathrm{RF}} m N e^{2(\mu-u)}}{\sigma^{2}}\left(e^{u-\mu}-e^{u}\right)-1}{\frac{P_{\mathrm{RF}} \sqrt{2 m N} e^{(\mu-u)}}{\sigma}}\right)-\operatorname{erf}\left(\frac{\frac{P_{\mathrm{RF}} m N e^{2(\mu-u)}}{\sigma^{2}}\left(e^{u-\mu}-1\right)-1}{\frac{P_{\mathrm{RF}} \sqrt{2 m N} e^{(\mu-u)}}{\sigma}}\right)\right) \mathcal{V}(u) .
\end{aligned}
$$

outage probability, we use (10) to upper bound the probability terms $F_{W_{m}}(u), \forall m$, by

$$
\begin{aligned}
& F_{W_{m}}(u) \stackrel{(i)}{=} Q\left(\frac{\sqrt{m N}(\mu-u)}{\sigma}\right)-e^{-\frac{e^{u}-1}{P_{\mathrm{RF}}}} Q\left(\frac{\sqrt{m N} \mu}{\sigma}\right) \\
& -\frac{P_{\mathrm{RF}} \sqrt{m N}}{\sqrt{2 \pi} \sigma} \int_{0}^{\frac{e^{u}-1}{P_{\mathrm{RF}}}} \frac{e^{-x}}{1+P_{\mathrm{RF}} x} e^{-\frac{m N\left(\log \left(1+P_{\mathrm{RF}} x\right)+\mu-u\right)^{2}}{2 \sigma^{2}}} \mathrm{~d} x \\
& \stackrel{(j)}{\leq} Q\left(\frac{\sqrt{m N}(\mu-u)}{\sigma}\right)-e^{-\frac{e^{u}-1}{P_{\mathrm{RF}}}} Q\left(\frac{\sqrt{m N} \mu}{\sigma}\right) \\
& -\frac{\sqrt{m N} e^{\frac{1}{P_{\mathrm{RF}}}-\frac{-m N \epsilon\left(\mu-u-\frac{\epsilon}{2}\right)}{\sigma^{2}}}}{\sqrt{2 \pi} \sigma} \int_{1}^{e^{u}} \frac{e^{-\frac{t}{P_{\mathrm{RF}}}}}{t^{1+\frac{m N \epsilon}{\sigma^{2}}}} \mathrm{~d} t \\
& =Q\left(\frac{\sqrt{m N}(\mu-u)}{\sigma}\right)-e^{-\frac{e^{u}-1}{P_{\mathrm{RF}}}} Q\left(\frac{\sqrt{m N} \mu}{\sigma}\right) \\
& -\frac{\sqrt{m N} e^{\frac{1}{P_{\mathrm{RF}}}-\frac{-m N \epsilon\left(\mu-u-\frac{\epsilon}{2}\right)}{\sigma^{2}}}}{\sqrt{2 \pi} \sigma} \times \\
& \left(E_{1+\frac{m N \epsilon}{\sigma^{2}}}\left(\frac{1}{P_{\mathrm{RF}}}\right)-e^{-\frac{m N \epsilon u}{\sigma^{2}}} E_{1+\frac{m N \epsilon}{\sigma^{2}}}\left(\frac{e^{u}}{P_{\mathrm{RF}}}\right)\right)=\mathcal{T}(u) .
\end{aligned}
$$

In (18), $(i)$ is based on partial integration. Also, $(j)$ follows from $(a-b)^{2} \geq \max \left(0,2 a \epsilon-2 b \epsilon-\frac{\epsilon^{2}}{2}\right), \forall a \geq b, \epsilon \geq 0$, and variable transform $t=1+P_{\mathrm{RF}} x$. Finally, the last equality is obtained by manipulations and the definition of the $n$-th order exponential integral function $E_{n}(x)=\int_{1}^{\infty} t^{-n} e^{-t x} \mathrm{~d} t$. Note that the bound is reasonably tight for different values of $\epsilon \geq 0$. Then, the appropriate value of $\epsilon$ can be determined numerically such that the difference between the exact and the bounded probabilities is minimized.

In Section $\mathrm{V}$, we validate the accuracy of the bounds/approximations proposed in (4)-(18) by comparing them with the corresponding values obtained via simulations. Finally, it is interesting to note that 1) as previously proved in [18, Section V.B], the same performance is achieved by the INR and repetition time diversity (RTD) HARQ systems at low SNRs. Thus, although the paper concentrates on the INR HARQ, the same conclusions hold for the RTD-based HARQ setups, as long as the SNR is low. Also, 2) the paper concentrates on the RF-FSO based systems. However, the same system model as in Figs. 1-2 holds in various coordinated data transmission schemes, for which the analytical results of Section IV are useful.

\section{Simulation Results}

Throughout the paper, we presented different approximation/bounding techniques. The verification of these results is demonstrated in Figs. 3-4 and, as seen in the sequel, the analytical results follow the simulations with high accuracy. Then, to avoid too much information in each figure, Figs. 5-6 report only the simulation results. Note that in all simulations we have double-checked the results with the ones obtained analytically. Moreover, in all figures, we consider uniform power allocation between the RF and the FSO links, i.e., $P_{\mathrm{RF}}=P_{\mathrm{FSO}}=\frac{P}{2}$. Hence, the sum power is $P$ (in $\mathrm{dB}$, $10 \log _{10} P$ ) which, because the noise variances are set to 1, is referred to as the SNR as well. In Figs. 3-5, we assume the FSO link to follow the exponential distribution $f_{G_{\mathrm{FSO}}}(x)=\lambda_{\mathrm{FSO}} e^{-\lambda_{\mathrm{FSO}} x}$ with $\lambda_{\mathrm{FSO}}=1$ or the log-normal distribution $f_{G_{\mathrm{FSO}}}(x)=\frac{1}{\sqrt{2 \pi} \delta x} e^{-\frac{(\log (x)-\varpi)^{2}}{2 \delta^{2}}}$ with $\delta=1$ and $\varpi=0$. In Fig. 6, the RF and the FSO links are supposed to experience exponential distributions $f_{G_{\mathrm{RF}}}(x)=\lambda_{\mathrm{RF}} e^{-\lambda_{\mathrm{RF}} x}$ and $f_{G_{\mathrm{FSO}}}(x)=\lambda_{\mathrm{FSO}} e^{-\lambda_{\mathrm{FSO}} x}$, where $\lambda_{\mathrm{RF}}$ and $\lambda_{\mathrm{FSO}}$ follow normalized log-normal distributions.

The simulation results are presented as follows.

On the bounding/approximation approaches of Lemmas 13: Setting $R=5 \mathrm{npcu}$ and $N=50$, Figs. 3-4 verify the tightness of the approximation/bounding schemes of Lemmas 1-3 for the exponential and log-normal distributions of the FSO link, respectively. As seen, the analytical results of Lemmas 1 


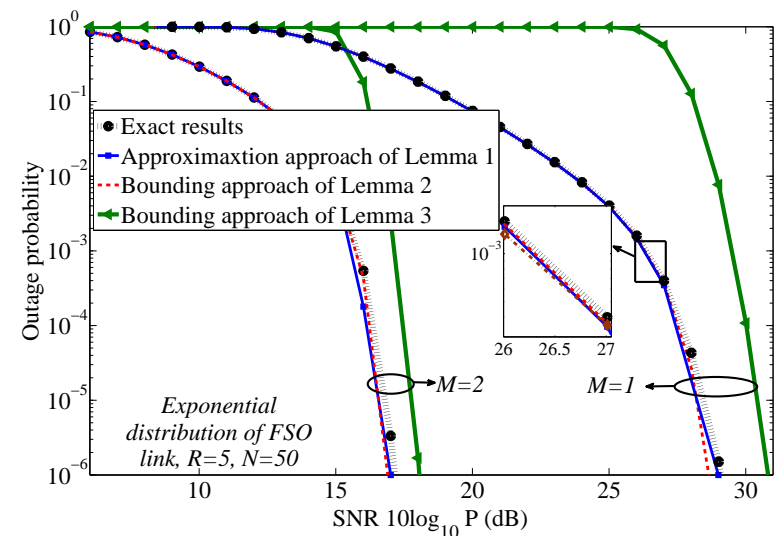

Figure 3. Comparison between the numerical and approximation results of Lemmas 1-3 (exponential distribution of the FSO link, $R=5$ and $N=50$ ).

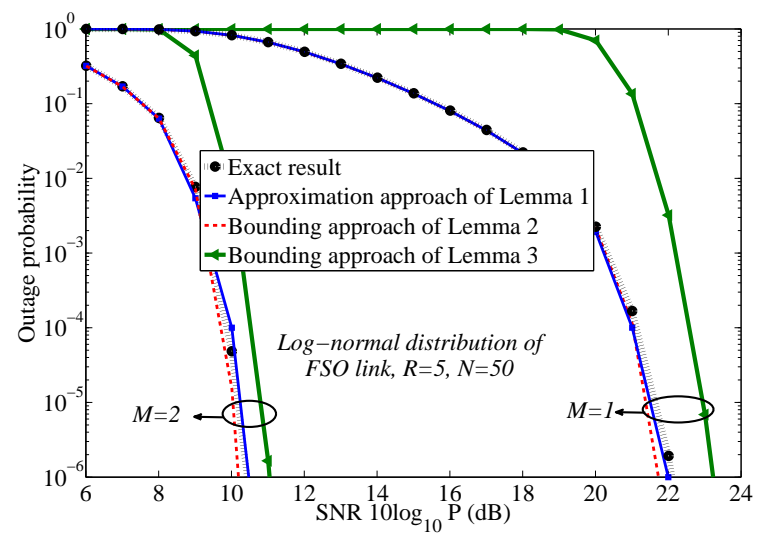

Figure 4. Comparison between the numerical and approximation results of Lemmas 1-3 (log-normal distribution of the FSO link, $R=5$ and $N=50$ ).

and 2 mimic the exact results with very high accuracy. Also, Lemma 3 properly upper-bounds the outage probability and the tightness increases with the SNR and/or number of retransmission rounds. Also, for a given number of retransmissions $M$, all curves follow the same diversity gain (slope of the curves at high SNRs). In this way, according to Figs. 3-4, the CLT-based approximation approaches of Lemmas 1-3 provide effective tools for the analytical investigation of the RF-FSO systems, if the links experience different coherence times.

On the effect of HARQ: Shown in Fig. 5 are the outage probability and throughput of the RF-FSO system for different maximum number of HARQ retransmission rounds $M$. Here, the results are presented for the exponential PDF of the FSO link, while the same trend is observed for the Gamma-Gamma [29] and log-normal PDFs of the FSO link as well. As shown in the figure, the implementation of HARQ leads to significant outage probability reduction at moderate/high SNRs. On the other hand, the HARQ is more useful, in terms of throughput, at low/moderate SNRs. However, at high SNRs and with given rates, the effect of HARQ on the throughput becomes negligible, because the data is decoded successfully in the first retransmission(s) with high probability. Finally, for different distributions of the FSO link, the throughput increases with the maximum number of retransmissions $M$, and the largest rel-
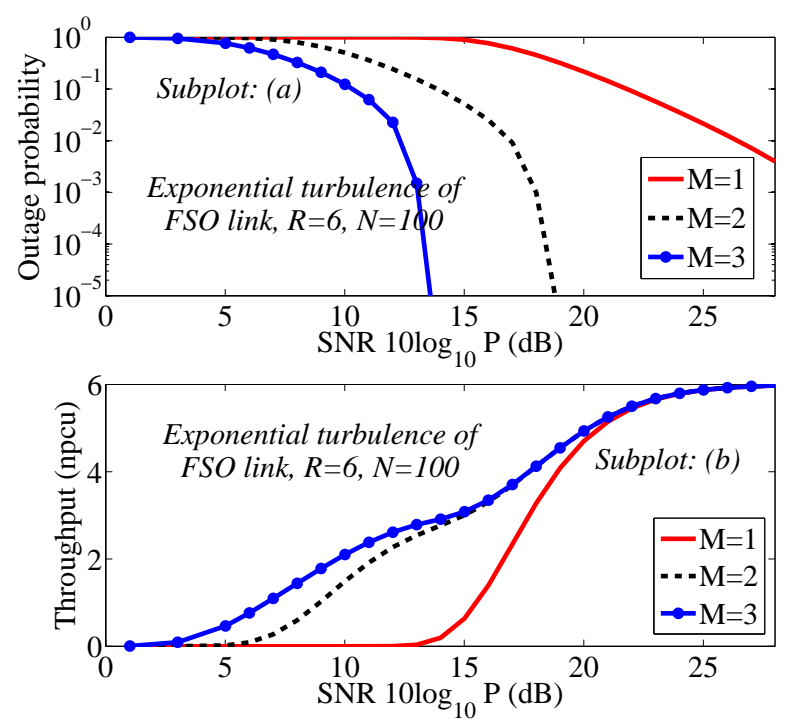

Figure 5. The effect of the number of HARQ retransmissions on the (a): outage probability, (b): throughput. Exponential PDF of FSO link, $f_{G_{\mathrm{FSO}}}(x)=\lambda e^{-\lambda x}, \lambda=1, R=6$ npcu and $N=100$.

ative throughput/outage probability improvement is observed when going from open-loop communication $(M=1)$ to the cases with a maximum of $M=2$ retransmissions.

Comparison between the performance of the RF, the FSO and the RF-FSO based systems: In Fig. 6, we compare the outage probability in the systems using only the RF link, only the FSO link and the joint RF-FSO transmission setup. Here, the results are obtained for the exponential PDFs of the RF and FSO links, i.e., $f_{G_{\mathrm{RF}}}(x)=\lambda_{\mathrm{RF}} e^{-\lambda_{\mathrm{RF}} x}$ and $f_{G_{\mathrm{FSO}}}(x)=\lambda_{\mathrm{FSO}} e^{-\lambda_{\mathrm{FSO}} x}$, where $\lambda_{\mathrm{RF}}$ and $\lambda_{\mathrm{FSO}}$ follow normalized log-normal distributions. Also, to have a fair comparison, the transmission powers are set to $\left(P_{\mathrm{RF}}=P, P_{\mathrm{FSO}}=0\right)$, $\left(P_{\mathrm{RF}}=0, P_{\mathrm{FSO}}=P\right)$ and $\left(P_{\mathrm{RF}}=\frac{P}{2}, P_{\mathrm{FSO}}=\frac{P}{2}\right)$ in the cases with only RF, only FSO and RF-FSO system, respectively, such that the sum power remains the same in different cases.

As demonstrated, the RF-FSO link leads to substantially less outage probability, compared to the cases with only the RF or the FSO link. For instance, with the initial rate $R=5 \mathrm{npcu}$, $M=2$ retransmissions, and the outage probability $10^{-2}$, the joint RF-FSO based data transmission reduces the required power by 16 and $4 \mathrm{~dB}$, compared to the cases with only the RF or the FSO link, respectively. Intuitively, this is because with the joint RF-FSO setup the diversity increases and the RF (resp. the FSO) link compensate the effect of the FSO (resp. RF) link, if it experiences severe fading conditions. Also, the effect of the joint transmission increases with the number of retransmissions/SNRs (Fig. 6).

\section{CONCLUSION}

This paper studied the performance of RF-FSO systems in the cases with perfect CSI at the receivers. We derived closed-form expressions for the message decoding probabilities, throughput, and outage probability of the RF-FSO systems using HARQ. The results show that the joint im- 


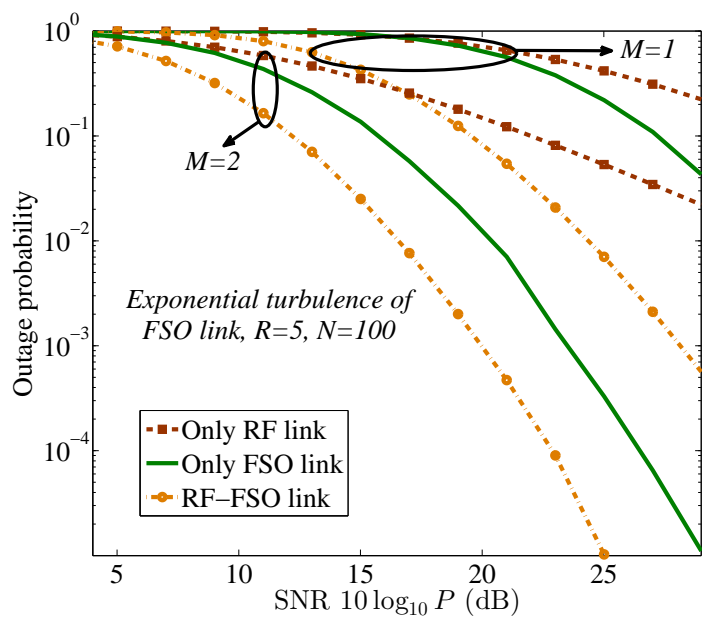

Figure 6. Comparison between the performance of the RF, the FSO, and the RF-FSO based systems. In all cases, the RF and the FSO links are supposed to follow $f_{G_{\mathrm{RF}}}(x)=\lambda_{\mathrm{RF}} e^{-\lambda_{\mathrm{RF}} x}$ and $f_{G_{\mathrm{FSO}}}(x)=\lambda_{\mathrm{FSO}} e^{-\lambda_{\mathrm{FSO}} x}$ where $\lambda_{\mathrm{RF}}$

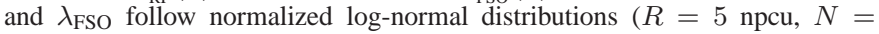
100).

plementation of RF and FSO links leads to considerable throughput and outage probability improvement, compared to the cases utilizing either the RF or the FSO link separately. Moreover, compared to open-loop communication, the HARQbased data transmission improves the throughput (resp. outage probability) of RF-FSO systems at low (resp. high) SNRs significantly. Among the interesting extensions of the paper is the performance analysis in the cases with the repetition time diversity and basic HARQ protocols.

\section{REFERENCES}

[1] A. Vavoulas, H. G. Sandalidis, and D. Varoutas, "Weather effects on FSO network connectivity," IEEE J. Opt. Commun. Netw., vol. 4, no. 10, pp. 734-740, Oct. 2012

[2] M. Usman, H. C. Yang, and M.-S. Alouini, "Practical switchingbased hybrid FSO/RF transmission and its performance analysis," IEEE Photon. J., vol. 6, no. 5, pp. 1-13, Oct. 2014

[3] H. Wu, B. Hamzeh, and M. Kavehrad, "Achieving carrier class availability of FSO link via a complementary RF link," in Proc. IEEE Asilomar'2004, vol. 2, California, USA, Nov. 2004, pp. 1483-1487 Vol.2.

[4] Z. Jia, F. Ao, and Q. Zhu, "BER performance of the hybrid FSO/RF attenuation system," in Proc. IEEE ISAPE'2006, Guilin, China, Oct. 2006, pp. 1-4.

[5] T. Kamalakis, I. Neokosmidis, A. Tsipouras, S. Pantazis, and I. Andrikopoulos, "Hybrid free space optical/millimeter wave outdoor links for broadband wireless access networks," in Proc. IEEE PIMRC'2007, Athens, Greece, Sept. 2007, pp. 1-5.

[6] H. Wu, B. Hamzeh, and M. Kavehrad, "Availability of airbourne hybrid FSO/RF links," in Proc. SPIE, 2005, vol. 5819

[7] Y. Tang and M. Brandt-Pearce, "Link allocation, routing and scheduling of FSO augmented RF wireless mesh networks," in Proc. IEEE ICC'2012, Ottawa, Canada, June 2012, pp. 3139-3143.

[8] A. Sharma and R. S. Kaler, "Designing of high-speed inter-building connectivity by free space optical link with radio frequency backup," IET Commun., vol. 6, no. 16, pp. 2568-2574, Nov. 2012.

[9] K. Kumar and D. K. Borah, "Hybrid FSO/RF symbol mappings: Merging high speed FSO with low speed RF through BICM-ID," in Proc. IEEE GLOBECOM'2012, California, USA, Dec. 2012, pp. 29412946.

[10] N. Letzepis, K. D. Nguyen, A. Guillen i Fabregas, and W. G. Cowley, "Outage analysis of the hybrid free-space optical and radio-frequency channel," IEEE J. Sel. Areas Commun., vol. 27, no. 9, pp. 1709-1719, Dec. 2009.
[11] S. Vangala and H. Pishro-Nik, "A highly reliable FSO/RF communication system using efficient codes," in Proc. IEEE GLOBECOM'2007, Washington, DC, USA, Nov. 2007, pp. 2232-2236.

[12] I. B. Djordjevic, B. Vasic, and M. A. Neifeld, "Power efficient LDPCcoded modulation for free-space optical communication over the atmospheric turbulence channel," in Proc. OFC/NFOEC, Anaheim, CA, USA, March 2007, pp. 1-3.

[13] B. He and R. Schober, "Bit-interleaved coded modulation for hybrid RF/FSO systems," IEEE Trans. Commun., vol. 57, no. 12, pp. 37533763, Dec. 2009.

[14] A. Abdulhussein, A. Oka, T. T. Nguyen, and L. Lampe, "Rateless coding for hybrid free-space optical and radio-frequency communication," IEEE Trans. Wireless Commun., vol. 9, no. 3, pp. 907-913, March 2010.

[15] P. Wu and N. Jindal, "Performance of hybrid-ARQ in block-fading channels: A fixed outage probability analysis," IEEE Trans. Commun., vol. 58, no. 4, pp. 1129-1141, April 2010.

[16] G. Caire and D. Tuninetti, "The throughput of hybrid-ARQ protocols for the Gaussian collision channel," IEEE Trans. Inf. Theory, vol. 47, no. 5, pp. 1971-1988, July 2001.

[17] H. E. Gamal, G. Caire, and M. O. Damen, "The MIMO ARQ channel: Diversity-multiplexing-delay tradeoff," IEEE Trans. Inf. Theory, vol. 52, no. 8, pp. 3601-3621, Aug. 2006.

[18] B. Makki and T. Eriksson, "On the performance of MIMO-ARQ systems with channel state information at the receiver," IEEE Trans. Commun., vol. 62 , no. 5, pp. 1588-1603, May 2014.

[19] D. Tuninetti, "On the benefits of partial channel state information for repetition protocols in block fading channels," IEEE Trans. Inf. Theory, vol. 57, no. 8, pp. 5036-5053, Aug. 2011.

[20] C. Shen and M. P. Fitz, "Hybrid ARQ in multiple-antenna slow fading channels: Performance limits and optimal linear dispersion code design," IEEE Trans. Inf. Theory, vol. 57, no. 9, pp. 5863-5883, Sept. 2011.

[21] B. Makki, T. Svensson, and M. Zorzi, "Finite block-length analysis of the incremental redundancy HARQ," IEEE Wireless Commun. Lett., vol. 3, no. 5, pp. 529-532, Oct. 2014

[22] S. M. Aghajanzadeh and M. Uysal, "Information theoretic analysis of hybrid-arq protocols in coherent free-space optical systems," IEEE Trans. Commun., vol. 60, no. 5, pp. 1432-1442, May 2012.

[23] _ "Outage analysis of hybrid-ARQ protocols in coherent free-space optical communications," in Proc. IEEE PIMRC'2011, Toronto, Canada, Sept. 2011, pp. 1773-1777.

[24] E. Zedini, A. Chelli, and M.-S. Alouini, "On the performance analysis of hybrid ARQ with incremental redundancy and with code combining over free-space optical channels with pointing errors," IEEE Photon. J., vol. 6, no. 4, pp. 1-18, Aug. 2014.

[25] C. Kose and T. R. Halford, "Incremental redundancy hybrid ARQ protocol design for FSO links," in Proc. IEEE MILCOM'2009, Boston, USA, Oct. 2009, pp. 1-7.

[26] K. Kiasaleh, "Hybrid ARQ for FSO communications through turbulent atmosphere," IEEE Commun. Lett., vol. 14, no. 9, pp. 866-868, Sept. 2010.

[27] A. R. Hammons and F. Davidson, "On the design of automatic repeat request protocols for turbulent free-space optical links," in Proc. IEEE MILCOM'2010, California, USA, Oct. 2010, pp. 808-813.

[28] J. Perez-Ramirez and D. K. Borah, "Design and analysis of bit selections in HARQ algorithm for hybrid FSO/RF channels," in Proc. IEEE VTC Spring'2013, Dresden, Germany, June 2013, pp. 1-5.

[29] B. Makki, T. Svensson, T. Eriksson, and M.-S. Alouini, "On the performance of RF-FSO links with and without hybrid arq," IEEE Trans. Commun., 2015, submitted, Available per request.

[30] T. J. Moulsley and E. Vilar, "Experimental and theoretical statistics of microwave amplitude scintillations on satellite down-links," IEEE Trans. Antennas Propag., vol. 30, no. 6, pp. 1099-1106, Nov. 1982.

[31] C. E. Mayer, B. E. Jaeger, R. K. Crane, and X. Wang, "Ka-band scintillations: Measurements and model predictions," Proc. IEEE, vol. 85, no. 6, pp. 936-945, Jun. 1997.

[32] M.-S. Alouini, S. A. Borgsmiller, and P. G. Steffes, "Channel characterization and modeling for Ka-band very small aperture terminals," Proc. IEEE, vol. 85, no. 6, pp. 981-997, Jun. 1997.

[33] A. El Gamal and Y.-H. Kim, "Lecture notes on network information theory," 2010, [Online]. Available at http://arxiv.org/abs/1001.3404v4/.

[34] T. M. Cover and J. A. Thomas, Elements of Information Theory. New York: Wiley Interscience, 1992.

[35] L. C. Andrews and R. L. Phillips, Laser Beam Propagation through Random Media. 2nd ed. Bellingham, Washington, SPIE Press, 2005. 